\title{
Use of Assistance and Therapy Dogs for Children with Autism Spectrum Disorders: A Critical Review of the Current Evidence
}

\author{
Alessandra Berry, PhD, Marta Borgi, MS, Nadia Francia, PhD, \\ Enrico Alleva, PhD, and Francesca Cirulli, PhD
}

\begin{abstract}
Background: Autism spectrum disorders (ASD) are characterized by deficits in social reciprocity and communication, and by unusually restricted, repetitive behaviors. Intervention strategies based on the exploitation of the emotional aspects of human-dog relationships hold the potential to overcome the difficulty of subjects with ASD to relate and interact effectively with others, targeting core symptoms of this disorder.

Methods: This review summarizes the results of six published studies on the effects of brief interactions with dogs and the effects of introducing dogs in families with a child diagnosed with ASD, with an emphasis on social behaviors and language use. Furthermore, the possible mechanisms responsible for the beneficial effects observed are discussed.

Conclusions: Although the studies described here are encouraging, further research with better designs and using larger samples is needed to strengthen translation of such interventions to the clinic. In addition, potential applications of analyzing child-dog interactions are highlighted to screen for early signs of the disorder.
\end{abstract}

\section{Introduction}

I N 1943, LEO KANNER FIRST DESCRIBED children who lacked interest in other people, using the term "early infantile autism." One (1) year later, Hans Asperger independently reported of a group of children characterized by similar behavioral features but with milder severity and higher intellectual abilities. ${ }^{1}$

Autism is the prototypical form of a spectrum of related, complex, neurodevelopmental disorders referred to as autistic spectrum disorders (ASDs), also known as pervasive developmental disorders (PDDs). These include autism, Asperger syndrome, and atypical autism, ${ }^{2}$ and it has been estimated that they affect 1 child in about 150 children, $^{3}$ although several factors may lead to an underestimation of the prevalence. ${ }^{4}$ ASDs are strongly biased toward males (with a male/female ratio of 4:1 for classic autism), suggesting a potential involvement of perturbations in the typical trajectory and maturation of the sexually dimorphic brain in the etiology of this disease. ${ }^{3,5,6}$ Impairments in reciprocal social interaction and deficits in age-specific play patterns and symbolic behavior are key features indicative of autism in infants and preschoolers. In particular, a diagnosis of autism, based on the Diagnostic and Statistical Manual of Mental
Disorders (DSM IV) and on the International Statistical Classification of Diseases and Related Health Problems, 2,7 implies impairments in three behavioral domains: (1) social interaction; (2) language, communication, and imaginative play; and (3) restricted range of interests and activities. The onset of the disease must be before the age of 3 years.

Autism is recognized as a neurobiological condition involving central nervous system dysfunctions with a strong genetic basis, involving multiple, interacting genes, as confirmed from twin and family studies. ${ }^{8-11}$ However, the precise etiology of ASD still remains unknown because of the complex behavioral phenotypes and multigenic factors characterizing this disorder. In addition, exposure to environmental modifiers might contribute to variable expression of autism-related traits. ${ }^{12}$ Indeed, recent studies suggest that programming effects on the developing offspring, resulting from stressful experiences, maternal diet and infections, advanced parental age, prematurity, and low birth weight-just to mention a few-might act through epigenetic mechanisms resulting in critical determinants

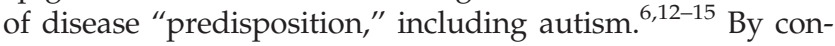
trast, intensive behavioral interventions carried out during early childhood have been shown to decrease symptom severity. ${ }^{16,17}$ 
A growing number of therapeutic approaches exist for the management of infantile autism. ${ }^{16,18}$ Some of these involve developmental/behavioral strategies; others are based on sensory-integration or social skills training, whereas pharmacologic therapies are not specifically meant to target the core symptoms of ASD, but rather to act on some psychiatric aspects associated with this pathology (hyperactivity, inattention, aggression, irritability, and self-injurious behaviors). ${ }^{16,19-24}$ Intervention programs based on behavioral strategies, such as the Lovaas program, ${ }^{25}$ have shown to successfully improve communication, social skills, and management of "problematic" behaviors, and currently represent the most effective methods for ASD management. ${ }^{26}$ However, despite the large number of therapeutic approaches, at present, neither proven therapies nor preventive measures exist for the universal treatment of infantile autism. Therefore, further research and the development of individualized therapies for the ASD population appear imperative.

It is possible to hypothesize that intervention strategies based on exploiting the emotional aspects of the relationship with a dog might represent an effective tool to dampen withdrawal of children with ASD by targeting some of the core symptoms of this disorder. The simple and interpretable pattern of movements that characterizes dogs might facilitate the engagement of children with ASD in structurally simple social actions that do not require the interpretation of verbal cues and are highly repeatable and predictable (e.g., throw, fetch and retrieve play, walking the dog on a leash, giving a hand command). ${ }^{27-29}$ Sams and colleagues suggest that acquiring the ability to interpret and respond to the social and behavioral cues of dogs may provide a bridge toward learning to interpret the more subtle behavior of human beings. ${ }^{28}$ It has also been suggested that dogs, representing a powerful multisensory stimulus-strong and clear sounds, a vivid visual impression, a special smell, and a stimulation to touch-might target the low sensory and affective arousal levels characterizing children with ASD. ${ }^{29}$ In this context, it is worth noticing that the Denver Model, a therapeutic intervention that integrates applied behavior analysis with developmental and relationship-based approaches, ${ }^{30,31}$ involves the use of "sensory social routines" (i.e., repeated dyadic interactions, based on pleasurable activities, that have strong sensory, movement, and social foundations). ${ }^{19}$ Thus, dogs appear to be good candidates to assist therapists in building such pleasurable dyadic interactions.

Generally, dogs are receiving increasing attention because of their potential use in various therapeutic/activity programs (therapy dogs), also known as Animal-Assisted Interventions (AAI), aimed at targeting a large number of health-related problems in different clinical populations. ${ }^{32,33}$

Different reports have also shown the overall ability of assistance dogs (which are owned by individuals with different disabilities) to act as social catalysts, enhancing social interactions and increasing social networks, especially for those with severe disabilities, who very often suffer social discrimination. ${ }^{34-38}$

Despite the growing body of evidence showing the potential of interaction with dogs to benefit people, including those with disabilities, only a limited amount of studies have specifically focused on the role of child-dog relationships in the ASD population. In addition, most of these studies are lacking a standard methodology, making it difficult to evaluate the efficacy of the intervention.

The aim of this review is to provide a critical and systematic reading of the literature assessing the effects of therapy and assistance dogs on children with ASD and the possible overall reflection on the welfare of their families.

\section{Targeting Autism Through the Interaction with Dogs: A Review of the Literature}

Although the first reports on the beneficial effects of dogs with severely withdrawn children date back to the 1960s with the experience of Boris Levinson, ${ }^{39}$ successive studies aimed at evaluating the efficacy of systematic interactions of children affected by ASD with dogs are scarce. In the late 1980 s, Redefer and Goodman ${ }^{29}$ were pioneers in the scientific approach of the effects of interaction with dogs on children with autism, although a systematic review of the literature revealed that it was only from 2000 that this field of research has been receiving growing attention, as reflected in the increasing amount of studies published.

For the purpose of this review, only experimental studies, semistructured interviews, and case studies, published in the English language in peer-reviewed journals, have been taken into account. Qualitative (anecdotal) studies were excluded, as well as published $\mathrm{PhD}$ theses and conference proceedings. The literature search included the following databases: PubMed, Google Scholar, ScienceDirect, and ERIC. Reference lists in studies and review articles about this intervention were also consulted to identify potentially relevant studies. Participants were children (age range: 3-15) diagnosed with a PDD, according to DSM-IV. Research that did not entirely focus on autistic disorders (i.e., large range of clinical syndromes, small number of people with autism) was excluded. Interventions involving therapy were distinguished from those involving assistance dogs. As for therapy dogs, interactions occurred in the presence of the therapist, who followed a predetermined research protocol that included structured oneto-one activities designed to stimulate social behaviors and language use. Assistance dogs were introduced into the families with the primary function to ensure child safety, at home and in public, by inhibiting children from bolting or moving dangerously (e.g., walking off the sidewalk). ${ }^{40}$

Results of this literature search revealed that only six articles met the inclusion criteria: two of them describe the effects of assistance dogs (Table 1) and four interventions involve the effects of therapy dogs (Table 2) on children with PDD, including ASD.

\section{Effects of Interventions}

\section{Assistance dogs}

When studying the effects of the introduction of assistance dogs on the general welfare of families with children affected by ASD, Burrows and colleagues ${ }^{41}$ observed that the contribution of these animals extended beyond children's physical safety to therapeutic and communicative domains. In fact, the integration of assistance dogs resulted in beneficial effects on children's behavior with decreased anxiety and anger, increased calmness, reduction in the number of emotional outbursts (tantrums), and more manageable bedtime routines. These authors also report an additional sense 


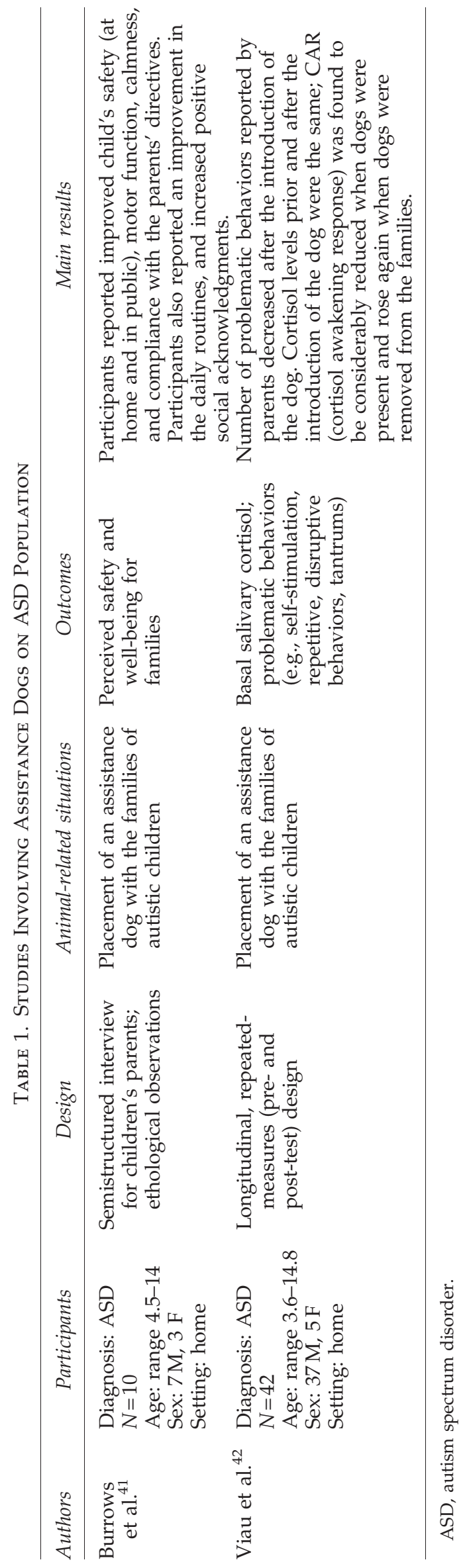

of security for the parents, especially at night, which improved their quality and quantity of sleep and their sense of independence. In addition, all the members of the family experienced an increase in the social acknowledgment perceived, thanks to the presence of the assistance dog. In fact, the dog became the focus of attention, alleviating some of the embarrassment and stress felt by the parents and the siblings of the autistic child and helping the whole family to integrate into the community. ${ }^{41}$

A different study focused on the specific effect of assistance dogs in regulating a physiologic response to stress in autistic children. Viau and colleagues ${ }^{42}$ measured physiologic variables (i.e., cortisol levels) before, during the staying of the dogs, as well as after they were removed from the families. Authors found that the cortisol awakening response (CAR) was decreased upon the introduction of dogs (acute effect), whereas it rose again when the animals were removed from the families (long-term effects). Indeed, these results show that, in children with ASD, CAR is sensitive to the presence of assistance dogs, suggesting that this measure might represent a reliable physiologic marker of a positive/ negative psychologic state. Interestingly, parents also reported a decrease in the number of problematic behaviors (such as self-stimulation, repetitive behaviors, and tantrums) when the dogs were living in the families, which lends support to the potential behavioral benefits of assistance dogs for children with ASD. ${ }^{42}$

It is important to stress that, in both of these studies, the effect of an assistance dog on children with problematic behaviors was assessed by means of semistructured interviews and a questionnaire distributed to parents, possibly reflecting the parents' satisfaction more than an actual modification in their child's behavior. The analysis of behavioral changes based on systematic observations might represent a more useful and comprehensive approach.

\section{Therapy dogs}

Interventions with therapy dogs rely upon brief interactions with these animals (AAI). All the interventions reviewed here occurred in the presence of the therapist, who followed a predetermined research protocol that included structured one-to-one (individual) activities designed to stimulate social behaviors and language use. In all cases, results were encouraging since interaction with dogs was able to dampen social isolation and withdrawal in children.

Redefer and Goodman ${ }^{29}$ observed that, following the introduction of a friendly dog into a therapeutic session, seriously withdrawn children with ASD showed a sharp increase in the frequency of both verbal and nonverbal social behaviors; this increase was directed toward the dog and the therapist and was paralleled by a decrease in children's withdrawal. This general improvement could still be observed 1 month after dog exposure (follow-up), although in a smaller proportion. Limitations of this study are the lack of information about the diagnostic criteria used and the absence of a control condition, which permit singling out whether the positive effects observed during AAI are simply due to the introduction of a novel and exciting stimulus (e.g., a visiting dog).

Martin and Farnum ${ }^{43}$ observed children's social behavior when they were interacting with a live dog and when 


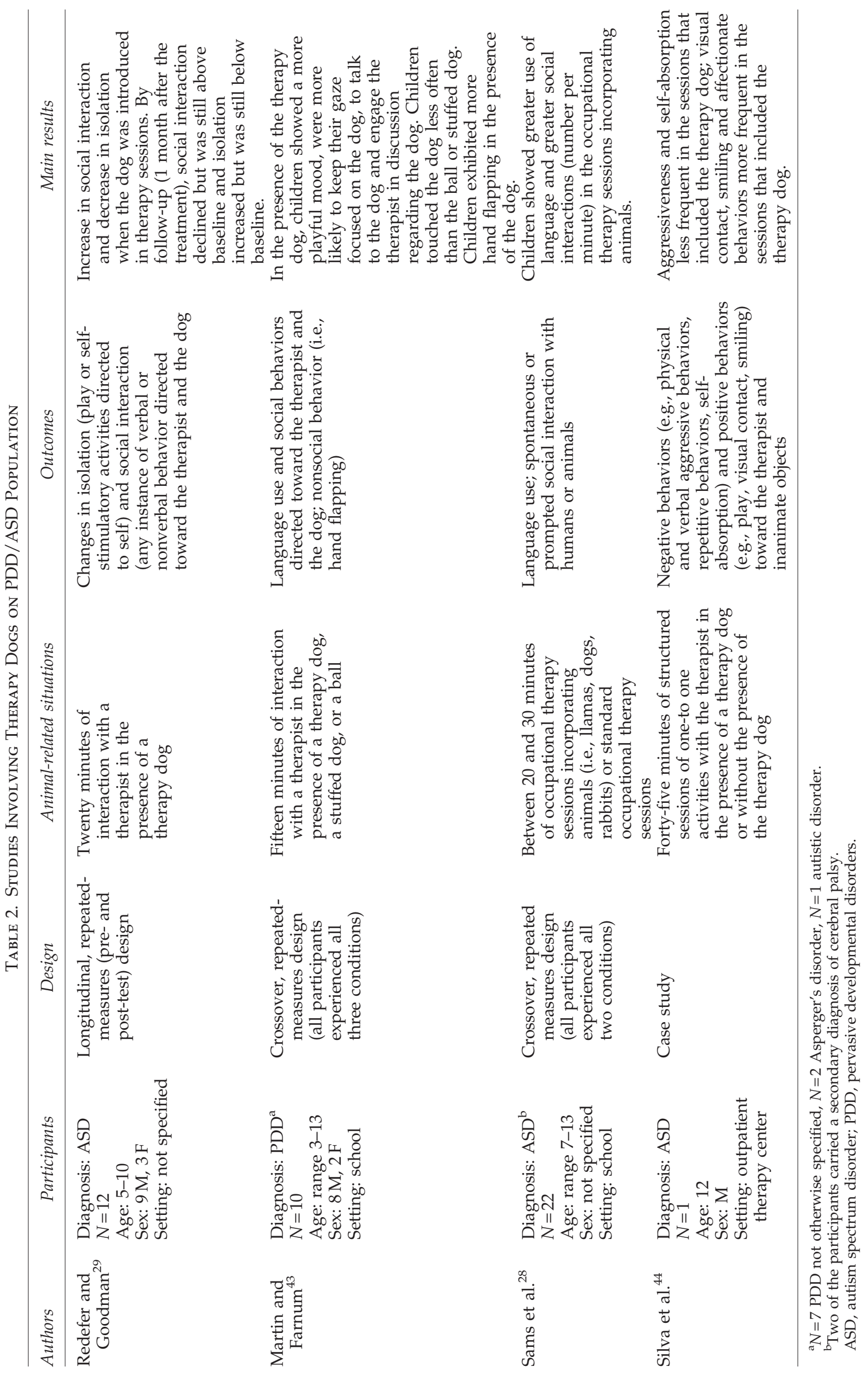


playing either with a nonsocial toy (i.e., a ball) or a stuffed dog. These authors found that children were less distracted, exhibited a more playful mood, and were more aware of their social environment when in the presence of the therapy dog. ${ }^{43}$ Furthermore, verbal interactions were stimulated by the presence of the animal: Children were more likely to talk to the dog, engaging the therapist in discussions regarding the animal, and speaking less about topics unrelated to the therapeutic protocol during the dog condition. An increase in hand flapping was also reported in children exposed to the therapy dog. Although such a behavior is usually considered as a stereotypy and therefore viewed as undesirable, the authors believe that this "was a mode of expressing excitement and exhilaration." ${ }^{\prime 43}$ It is worth noticing that this study represents a first important attempt to perform a thorough analysis of the effects of AAI with a dog through the comparison with behavioral changes resulting from the exposure to different stimuli (ball/stuffed dog).

Similar results were obtained by Sams and colleagues, ${ }^{28}$ who found that the incorporation of animals (i.e., llamas, dogs, and rabbits) in a school-based occupational therapy program was able to encourage language use and social interaction in children with ASD, particularly in comparison to occupational therapy using standard techniques.

The efficacy of the dogs' presence in increasing the engagement and decreasing negative behavioral patterns (such as aggressive and obsessive manifestations) was also confirmed in a very recent case-study by Silva and colleagues. ${ }^{44}$ In particular, physical and verbal aggressiveness, as well as self-absorption, were significantly reduced while smiles, visual contacts, and affectionate behaviors were increased. ${ }^{44}$

\section{Discussion}

By being able to respond affectionately to human attention, to elicit prosocial behavior and positive feelings, animals seem to possess a unique capacity to serve as an emotional bridge in specific therapeutic contexts and to act as social catalysts. ${ }^{39,45}$ The forms and manifestations of human bonds with companion animals, particularly with dogs, have led to their extensive inclusion in different educational and therapeutic interventions. ${ }^{32,33}$ In this context, attitude toward animals is thought to be sufficient to explain some of the beneficial effects observed, since things that tend to absorb people's attention in nonthreatening ways are also known to exert a calming and de-arousing influence. ${ }^{46-48} \mathrm{~A}$ more relaxing environment might, in turn, increase people's responsiveness and willingness to communicate. In fact, different studies reported that, when a dog was present, children appeared more prone to engage with peers and adults. ${ }^{4-51}$

Taken together, the studies reviewed here are encouraging, since the interaction of children affected by ASD with therapy dogs was able to promote verbal and nonverbal behaviors, directed both towards the dog and the therapist. ${ }^{28,43,44}$ Some authors have suggested that being able to gain access to pets, or to talk about them, might represent a strong drive for children with ASD to increase social interactions with the therapist. ${ }^{28}$ In this context, it is important to stress that language impairment is one of the most pervasive symptoms characterizing children with ASD early during postnatal life, and thus being able to act upon such a domain is a main challenge of current behavioral therapies. However, it is also important to take into account that the observed effects on verbal and social skills might in some cases be related to symptom severity; however, none of the studies reviewed considered this variable.

The introduction of assistance dogs into the families of children with ASD allows investigation of the long-term effects of interactions with dogs. In particular, Viau and colleagues $^{42}$ found a decrease in problematic behaviors in addition to a specific reduction in a stress-related hormonal parameter (CAR) only during the time the dog was housed with the family. By contrast, when the dog was moved away, the CAR rose again, suggesting that this measure of neuroendocrine activation is very sensitive to the presence of the dog. These data are in line with a substantial body of evidence showing the overall "de-arousing effect" of humananimal interactions on human physiology. ${ }^{52-58}$

The study by Burrows and colleagues found that the introduction of assistance dogs resulted in beneficial effects not only on children's behaviors but also on the overall welfare of the family. Family members reported a sense of security and independence, a result particularly important when considering that the impulsivity, hyperactivity, irritability, and aggressiveness of children with ASD profoundly influence parents' everyday activities, which eventually result in extremely stressful and unsafe experiences for the whole family. ${ }^{41,59,60}$ Moreover, an increase in the social acknowledgment helped the whole family to feel integrated into the community. This is indeed a desirable result, considering that these families have to deal with social isolation and often experience elevated levels of pessimism and of depressive symptoms. ${ }^{60-64}$ Overall, these findings are not surprising and are in agreement with reports showing the ability of dogs to act as social catalysts, enhancing social interaction between people and increasing social networks, especially for those who have severe disabilities and who very often suffer social discrimination. $^{34-38}$

Despite these positive and encouraging results, a note of caution must be placed here. In particular, it has been reported that the presence of a dog might elicit an increase in undesirable stereotyped behaviors (such as the hand-flapping $^{43}$ ), which are thought to attenuate or intensify the arousal levels of children with ASD, depending upon the environmental circumstances. ${ }^{65,66}$ Almost all individuals affected by autism are characterized by hyper- or hyporesponsiveness to sensory inputs ${ }^{65,67,68}$; therefore, stimuli that are experienced as neutral by typically developing children might result either in highly rewarding or aversive responses for children with ASD. ${ }^{69}$ Thus, in the case of assisted interventions with dogs, these might involve individualized programs tailored to each child's profile, taking into special account sensory difficulties and arousal levels. ${ }^{6,67}$

In conclusion, intervention strategies, based on exploiting the emotional aspects of the relationship with a dog, can overcome the inability of children affected by ASD to relate and interact with others by targeting some of the core symptoms of this disorder. However, it is important to take into account that overall most of the AAI programs lack a standard methodology, and there is a need for basic research aimed at including larger sample sizes to assess their effectiveness, using randomized controlled trial designs. In addition, studies specifically aimed to examine whether the 
effects of contact with dogs are enduring or are strictly related to a continuous exposure to the animal are still lacking. The use of systematic, detailed studies of behavior should be encouraged since they can better clarify the effectiveness of dog-oriented programs on children' behavior and overall quality of family life.

\section{Future Perspectives: Child-Dog Interaction as a New Screening Tool for Early ASD Diagnosis}

A diagnosis of autism is achieved early during childhood, with symptoms becoming manifest within the first 3 years of life. However, parents often recall having had developmental concerns between the first and the second birthdays of their children. This delay in the diagnosis prevents the possible benefits of an early intervention during a time window characterized by higher brain and behavioral plasticity. ${ }^{16,70-73}$ A promising study reports that characteristic interaction patterns exist during child-dog encounters, and this may be effective to diagnose psychiatric disorders including autism. Patients demonstrated significant diagnosis-specific behavioral differences; in particular, autism patients looked at the dog for a considerably shorter time, showed longer phases of distancing behavior from the dog, and stroked it less than other diagnostic groups did (i.e., anxiety disorder, anorexia, bulimia). ${ }^{74}$ In this scenario, it is possible to hypothesize that the identification of specific behavioral patterns displayed during child-dog interactions might provide a novel additional tool for the early diagnosis of some ASD signs, such as deviation from typical attentive and social behaviors (gazes, smiles, directed vocalizations) and changes in posture and movements towards the dog.

\section{Acknowledgments}

Funding for this study was provided by the Italian Ministry of Health (Italia-USA project 11US/11), Istituto Superiore di Sanità (Research project "Effects of dog-assisted therapies on physical and psychological well being in the institutionalized elderly"), and the Azienda per i Servizi Sanitari n. 6 Friuli Occidentale, collaborative project "Validazione di attività innovative in ambito agricolo finalizzate all'inserimento sociale, riabilitativo e lavorativo di persone diversamente abili."

\section{Disclosure Statement}

No competing financial interests exist.

\section{References}

1. Kolvin I. Studies in the childhood psychoses. I. Diagnostic criteria and classification. Br J Psychiatry 1971;118:381-384.

2. American Psychiatric Association. Diagnostic and Statistical Manual of Mental Disorders (DSM-IV). Washington: American Psychiatric Association, 1994.

3. Fombonne E. Epidemiology of pervasive developmental disorders. Pediatr Res 2009;65:591-598.

4. Lord C. Epidemiology: How common is autism? Nature 2011;474:166-168.

5. Baron-Cohen S, Lombardo MV, Auyeung B, et al. Why are autism spectrum conditions more prevalent in males? PLoS Biol 2011;9:e1001081.
6. Bale TL, Baram TZ, Brown AS, et al. Early life programming and neurodevelopmental disorders. Biol Psychiatry 2010;68: 314-319.

7. World Health Organization. International Classification of Diseases: Diagnostic Criteria for Research, 10th ed. Geneva: World Health Organization, 1993.

8. Abrahams BS, Geschwind DH. Advances in autism genetics: On the threshold of a new neurobiology. Nature Rev 2008; 9:341-355.

9. O'Roak BJ, State MW. Autism genetics: Strategies, challenges, and opportunities. Autism Res 2008;1:4-17.

10. State MW. The genetics of child psychiatric disorders: Focus on autism and Tourette syndrome. Neuron 2010;68:254-269.

11. Veenstra-Vanderweele J, Christian SL, Cook EH, Jr. Autism as a paradigmatic complex genetic disorder. Ann Rev Genom Hum Genetics 2004;5:379-405.

12. Muhle R, Trentacoste SV, Rapin I. The genetics of autism. Pediatrics 2004;113:e472-e486.

13. Patterson PH. Maternal infection and immune involvement in autism. Trends Molec Med 2011;17:389-394.

14. Scherer SW, Dawson G. Risk factors for autism: Translating genomic discoveries into diagnostics. Hum Genetics 2011; 130:123-148.

15. Shelton JF, Tancredi DJ, Hertz-Picciotto I. Independent and dependent contributions of advanced maternal and paternal ages to autism risk. Autism Res 2010;3:30-39.

16. Rogers SJ, Vismara LA. Evidence-based comprehensive treatments for early autism. J Clin Child Adolesc Psychol 2008;37:8-38.

17. Myers SM, Johnson CP. Management of children with autism spectrum disorders. Pediatrics 2007;120:1162-1182.

18. Reichow B. Overview of meta-analyses on early intensive behavioral intervention for young children with autism spectrum disorders. J Autism Dev Disord 2012;42:512-520.

19. Rogers S. Neuropsychology of autism in young children and its implications for early intervention. Mental Retard Dev Disabil Res Rev 1998;4:104-112.

20. Baranek GT. Efficacy of sensory and motor interventions for children with autism. J Autism Dev Disord 2002;32:397-422.

21. Cotugno AJ. Social competence and social skills training and intervention for children with autism spectrum disorders. J Autism Dev Disord 2009;39:1268-1277.

22. Granpeesheh D, Tarbox J, Dixon DR. Applied behavior analytic interventions for children with autism: A description and review of treatment research. Ann Clin Psychiatry 2009; 21:162-173.

23. Ospina MB, Krebs Seida J, Clark B, et al. Behavioural and developmental interventions for autism spectrum disorder: A clinical systematic review. PloS One 2008;3:e3755.

24. Leskovec TJ, Rowles BM, Findling RL. Pharmacological treatment options for autism spectrum disorders in children and adolescents. Harvard Rev Psychiatry 2008;16:97-112.

25. Lovaas OI. Behavioral treatment and normal educational and intellectual functioning in young autistic children. J Consult Clin Psychol 1987;55:3-9.

26. Vismara LA, Rogers SJ. Behavioral treatments in autism spectrum disorder: What do we know? Annu Rev Clin Psychol 2010;6:447-468.

27. Solomon $\mathrm{O}$. What a dog can do: Children with autism and therapy dogs in social interaction. ETHOS J Society Psychol Anthropol 2010;38:143-166.

28. Sams MJ, Fortney EV, Willenbring S. Occupational therapy incorporating animals for children with autism: A pilot investigation. Am J Occup Ther 2006;60:268-274. 
29. Redefer LA, Goodman JF. Brief report: Pet-facilitated therapy with autistic children. J Autism Dev Disord 1989;19: 461-467.

30. Rogers S, Herbison J, Lewis H, et al. An approach for enhancing the symbolic, communicative, and interpersonal functioning of young children with autism and severe emotional handicaps. J Div Early Child 1986;10:135-148.

31. Smith M, Rogers S, Dawson G. The Early Start Denver Model: A comprehensive early intervention approach for toddlers with autism. In: Handleman JS, Harris SL, eds. Preschool Education Programs for Children with Autism. Austin: Pro-Ed Corporation, 2008:65-101.

32. Friedmann E, Son H. The human-companion animal bond: How humans benefit. Vet Clin North Am 2009; 39:293-326.

33. Cirulli F, Borgi M, Berry A, et al. Animal-Assisted-Interventions as innovative tools for mental health. Ann Istit Superiore Sanità 2011;47:341-348.

34. Mader B, Hart LA, Bergin B. Social acknowledgments for children with disabilities: Effects of service dogs. Child Dev 1989;60:1529-1534.

35. Eddy J, Hart LA, Boltz RP. The effects of service dogs on social acknowledgments of people in wheelchairs. J Psychol 1988;122:39-45.

36. Davis BW, Nattrass K, O'Brien S, et al. Assistance dog placement in the pediatric population: Benefits, risks, and recommendations for future application. Anthrozoös 2004; 17:130-145.

37. Camp MM. The use of service dogs as an adaptive strategy: A qualitative study. Am J Occup Ther 2001;55:509-517.

38. Allen K, Blascovich J. The value of service dogs for people with severe ambulatory disabilities: A randomized controlled trial. JAMA 1996;275:1001-1006.

39. Serpell J. In the Company of Animals: A Study of HumanAnimal Relationships. Cambridge: Cambridge University Press, 1996.

40. Burrows KE, Adams CL. Challenges of service-dog ownership for families with autistic children: Lessons for veterinary practitioners. J Vet Med Educ 2008;35:559-566.

41. Burrows K, Adams C, Spiers J. Sentinels of safety: Service dogs ensure safety and enhance freedom and well-being for families with autistic children. Qual Health Res 2008;18: 1642-1649.

42. Viau R, Arsenault-Lapierre G, Fecteau S, et al. Effect of service dogs on salivary cortisol secretion in autistic children. Psychoneuroendocrinology 2010;35:1187-1193.

43. Martin F, Farnum J. Animal-assisted therapy for children with pervasive developmental disorders. West J Nurs Res 2002;24:657-670.

44. Silva K, Correia R, Lima M, et al. Can dogs prime autistic children for therapy? Evidence from a single case study. J Altern Complement Med 2011;17:655-659.

45. McNicholas J, Collis GM. Dogs as catalysts for social interactions: Robustness of the effect. Br J Psychol 2000;91:61-70.

46. Kellert SR. Affective, cognitive and evaluative perceptions of animals. In: Altman I, Wohlwill J, eds. Behavior and the Natural Environment. New York: Plenum Publishing Corporation, 1993:241-267.

47. DeSchriver M, Riddick C. Effects of watching aquariums on elders' stress. Anthrozoös 1990;4:44-48.

48. Katcher AH, Friedmann E, Beck AM, et al. Looking, talking and blood pressure: The physiological consequences of interaction with the living environment. In: Katcher $\mathrm{AH}$, Beck AM, eds. New Perspectives on Our Lives with Companion
Animals. Philadelphia: University of Pennsylvania Press, 1983:351-359.

49. Esteves WS, Stokes T. Social effects of a dog's presence on children with disabilities. Anthrozoös 2008;21:5-15.

50. Limond JA, Bradshaw JWS, Cormack KFM. Behavior of children with learning disabilities interacting with a therapy dog. Anthrozoös 1997;10:84-89.

51. Friesen L. Exploring animal-assisted programs with children in school and therapeutic contexts. Early Childhood Educ J 2010;37:261-267.

52. Odendaal JS, Meintjes RA. Neurophysiological correlates of affiliative behaviour between humans and dogs. Vet J 2003; 165:296-301.

53. Barker SB, Knisely JS, McCain NL, et al. Exploratory study of stress buffering response patterns from interaction with a therapy dog. Anthrozoös 2010;23:79-91.

54. Allen KM, Blascovich J, Tomaka J, et al. Presence of human friends and pet dogs as moderators of autonomic responses to stress in women. J Personality Soc Psychol 1991;61: 582-589.

55. Allen K, Shykoff BE, Izzo JL Jr. Pet ownership, but not ace inhibitor therapy, blunts home blood pressure responses to mental stress. Hypertension 2001;38:815-820.

56. Friedmann E, Locker B, Lockwood R. Perception of animals and cardiovascular responses during verbalization with an animal present. Anthrozoös 1993;6:115-134.

57. Friedmann E, Thomas S, Cook L, et al. A friendly dog as potential moderator of cardiovascular response to speech in older hypertensives. Anthrozoös 2007;20:51-63.

58. Allen K, Blascovich J, Mendes WB. Cardiovascular reactivity and the presence of pets, friends, and spouses: The truth about cats and dogs. Psychosom Med 2002;64: 727-739.

59. Phetrasuwan S, Shandor Miles M. Parenting stress in mothers of children with autism spectrum disorders. J Spec Pediatr Nurs 2009;14:157-165.

60. Abbeduto L, Seltzer MM, Shattuck P, et al. Psychological well-being and coping in mothers of youths with autism, Down syndrome, or fragile X syndrome. Am J Ment Retard 2004;109:237-254.

61. Briskin H, Liptak GS. Helping families with children with developmental disabilities. Pediatr Ann 1995;24:262-266.

62. Liptak GS, Orlando M, Yingling JT, et al. Satisfaction with primary health care received by families of children with developmental disabilities. J Pediatr Health Care 2006;20: 245-252.

63. Estes A, Munson J, Dawson G, et al. Parenting stress and psychological functioning among mothers of preschool children with autism and developmental delay. Autism 2009;13:375-387.

64. Sivberg B. Family system and coping behaviors: A comparison between parents of children with autistic spectrum disorders and parents with non-autistic children. Autism 2002;6:397-409.

65. Rogers SJ, Ozonoff S. Annotation: What do we know about sensory dysfunction in autism? A critical review of the empirical evidence. J Child Psychol Psychiatry Allied Discipl 2005;46:1255-1268.

66. Turner M. Annotation: Repetitive behaviour in autism. A review of psychological research. J Child Psychol Psychiatry Allied Discipl 1999;40:839-849.

67. Leekam SR, Nieto C, Libby SJ, et al. Describing the sensory abnormalities of children and adults with autism. J Autism Dev Disord 2007;37:894-910. 
68. Wiggins LD, Robins DL, Bakeman R, et al. Brief report: Sensory abnormalities as distinguishing symptoms of autism spectrum disorders in young children. J Autism Dev Disord 2009;39:1087-1091.

69. Klintwall L, Holm A, Eriksson M, et al. Sensory abnormalities in autism: A brief report. Res Dev Disabil 2011;32: 795-800.

70. Young RL, Brewer N, Pattison C. Parental identification of early behavioural abnormalities in children with autistic disorder. Autism 2003;7:125-143.

71. Ozonoff S, Iosif AM, Baguio F, et al. A prospective study of the emergence of early behavioral signs of autism. J Am Acad Child Adolesc Psychiatry 2010;49:256-266.

72. Gray KM, Tonge BJ. Are there early features of autism in infants and preschool children? J Paediatr Child Health 2001;37:221-226.
73. Dawson G. Early behavioral intervention, brain plasticity, and the prevention of autism spectrum disorder. Dev Psychopathol 2008;20:775-803.

74. Prothmann A, Albrecht K, Dietrich S, et al. Analysis of child-dog play behavior in child psychiatry. Anthrozoös 2005;18:43-58.

Address correspondence to: Francesca Cirulli, PhD

Section of Behavioural Neurosciences Department of Cell Biology and Neurosciences Istituto Superiore di Sanità Viale Regina Elena 299 Rome I-00161 Italy

E-mail: francesca.cirulli@iss.it 